\title{
LADM AND INDOORGML FOR SUPPORT OF INDOOR SPACE IDENTIFICATION
}

\author{
S. Zlatanova ${ }^{\mathrm{a}, *}$, P. J.M. Van Oosterom ${ }^{\text {a }}$, J. Lee ${ }^{\mathrm{b}}, \mathrm{K}^{-J}$ Li $^{\mathrm{c}}$, C.H.J. Lemmen ${ }^{\mathrm{d}}$ \\ ${ }^{a}$ Faculty of Architecture and the Built Environment, Delft University of Architecture, Julianalaan 134, 2628 BL, Delft, The \\ Netherlands - (s.zlatanova, p.j.m.vanoosterom)@tudelft.nl \\ b University of Seoul, Dongdaemoon-Gu, Seoul 02504, South Korea, jlee@uos.ac.kr \\ 'Pusan National University, Kumjeong-Gu, Pusan 46241, South Korea, lik@ pnu.edu \\ d Netherlands Cadastre, Land Registry and Mapping Agency (Kadaster), Chrit.Lemmen@kadaster.nl
}

Commission IV

KEY WORDS: Space subdivision, spatial units, rights, indoor, navigation, land administration, building model

\begin{abstract}
:
Guidance and security in large public buildings such as airports, museums and shopping malls requires much more information that traditional 2D methods offer. Therefore 3D semantically-reach models have been actively investigated with the aim to gather knowledge about availability and accessibility of spaces. Spaces can be unavailable to specific users because of plenty of reasons: the 3D geometry of spaces (too low, too narrow), the properties of the objects to be guided to a specific part of the building (walking, driving, flying), the status of the indoor environment (e.g. crowded, limited light, under reconstruction), property regulations (private areas), security considerations and so on.
\end{abstract}

However, such information is not explicitly avaible in the existing 3D semantically-reach models. IFC and CityGML are restricted to architectural building components and provide little to no means to describe such properties. IndoorGML has been designed to establish a generic approach for space identification allowing a space subdivision and automatic creation of a network for route computation. But currently it also represents only spaces as they are defined by the architectural layout of the building. The Land Administration Domain Model is currently the only available model to specify spaces on the basis of ownership and rights for use.

In this paper we compare the principles of IndoorGML and LADM, investigate the approaches to define spaces and suggest options to the linking of the two types of spaces. We argue that LADM space subdivision on basis of properties and rights of use can be used to define to semantically and geometrically available and accessible spaces and therefore can enrich the IndoorGML concept.

\section{INTRODUCTION}

Modelling of indoor spaces has been approached from two general points of view: the construction view and the user view. IFC (buildingSMART International, 2013) is one typical example of a building standard to describe internal building structures with focus on the construction elements (walls, slabs, doors, windows). Alternatively, CityGML LOD4 (Gröger et al., 2012) provides an indoor description from the view of 'being inside', i.e. describing the spaces (rooms, corridors) and neglecting the spaces occupied by building construction elements. These two standards have been also actively investigated to automatically derive a navigation network, but they appear insufficient for some specific environments (e.g. large rooms) or to serve certain navigation purposes (e.g. distinguish between walking and driving). Therefore, many models have been developed for the purpose of navigation. Among others, reviews of indoor models for navigation are presented by Bandi and Thalmann, 1998, Afyouni et al, 2012, Zlatanova et al 2013. Many frameworks for indoor space subdivision have been investigated as well (e.g. Raubal and Worboys 1999, Becker et al., 2008, Richter et al 2009, Worboys, 2011, Zlatanova et al 2013, Zlatanova et al 2014). The common shortcoming of these models is that they have been developed within the view of a specific application and/or target group of users.

* Corresponding author
The OCG standard IndoorGML (Lee et al., 2014, Li, 2016) is the first standard dedicated to provide a framework for operating indoor navigation systems. The standard is developed with the aim to reflect user specifications (Brown et al 2013, Zlatanova et al 2014) and provide a general framework for space definition (Figure 1). Prior IndoorGML, research has already indicated that a subdivision of indoor spaces according to human perception will improve the indoor guidance and navigation (e.g. Goetz and Zipf 2011, Kikiras, et al 2006). Based on the IndoorGML concept, Kruminaite and Zlatanova, 2014 proposed a set of criteria to define functional areas around interior furniture or other architectural elements. Sithole and Zlatanova, 2016 propose a framework for formalisation of human perceptions of position, location, place and area. But this research still relates to architectural characteristics of a building.

Defining accessible spaces and specifying security areas is often guided by legal restrictions or rights. Airports are typical example of establishing security areas on the basis of law-enforced subdivisions. Therefore, it is of great interest to investigate which types of rights or restriction have impact on the space subdivision. The Land Administration Data Model (LADM) is one of the few models, which aims at formally describing such information.

LADM is an ISO standard (ISO19152, 2012, Lemmen et al 2015, Van Oosterom and Lemmens 2015) and focuses on rights, 
restrictions and responsibilities outdoor and indoor including their geometrical representation, which might be defined by fictional boundaries. In general, land administration is a very broad and complex field and depends largely on the legislation of a specific country. Therefore, LADM tries to capture the principle and the common characteristics. This general view is very appropriate for the purpose of space subdivision in IndoorGML.

This paper compares the two models and recommends options to establish semantic and geometric relationship between spaces. The reminder of this paper is organised as follows: the next section provides details on the space subdivision according to IndoorGML. Section 3 introduces LADM and concentrates on classes influencing accessibility of spaces; e.g. legal spaces related to building units. Section 4 discusses several use cases and specifies options for linking IndoorGML and LADM. The last section summarises future research.

\section{INDOORGML}

IndoorGML distinguishes between two general spaces primal space and dual space (Figure 1). The primal space reflects the geometry, semantics and topology of the indoor space. The dual space is derived automatically from the primal space and used to define the Node-Relation Graph (NRG). Therefore, the space delineation is the most critical process. As it will be shown in the text below, spaces can be defined according to different criteria and can completely or partially ignore architectural boundaries. Distinct space subdivisions can be organised in the Multi-layered Space Model (MLSM) and used for integrated analysis in support of navigation or Location-Based Services (LBS) (
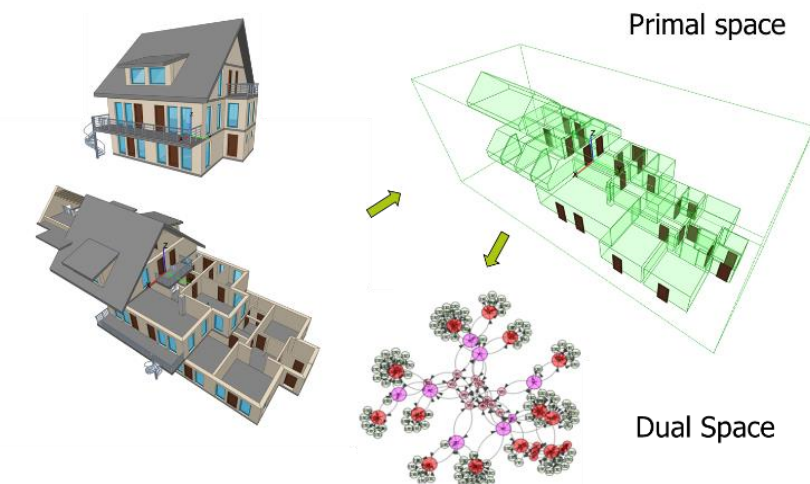

Figure 1: The general concept of IndoorGML: 3D model of a building (left); room (green) and door (brown) spaces used for navigation (right up); navigation network (right-down)

\subsection{Indoor space}

Indoor space consists of all cells that describe the architectural elements (rooms, walls, stairs, etc.) of a building. Indoor space can theoretically be defined according to different themes such as a building construction, functional use, security, sensor coverage, or a user mode of movement (e.g. walking, driving). A specific theme may require either subdivision or union of architectural elements (rooms and walls). For example. 'security area', 'check-in' area can be 'a part' of large hall, while 'dangerous area' can be a union of several rooms, or floors (Figure 2).
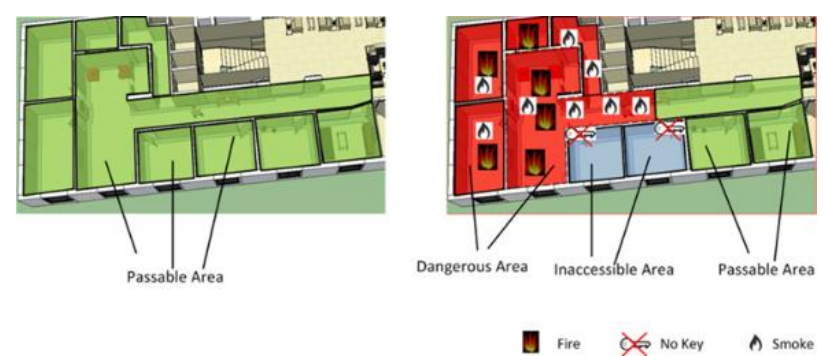

Figure 2: Union of topographic spaces to represent dangerous areas

The current version of IndoorGML focusses on so called 'topographic representation', which means the cells are defined with respect to the architectural subdivision of the building. The Indoor spaces of the current version of IndoorGML have the following characteristic:

- Navigable spaces include all architectural components (entrances, corridors, rooms, doors, and stairs), which are of importance for moving through the building. Construction elements are seen as obstacles and are clearly indicated as nonnavigable spaces. Furniture is not explicitly included, but it should be regarded non-navigable space.

- Spaces are closed objects, represented by areas in 2D and volumes in 3D. They may touch, but may not overlap.

- Spaces can be bordered by physical or fictional boundaries, or combinations of them.

- Spaces are semantically identified (Figure3):

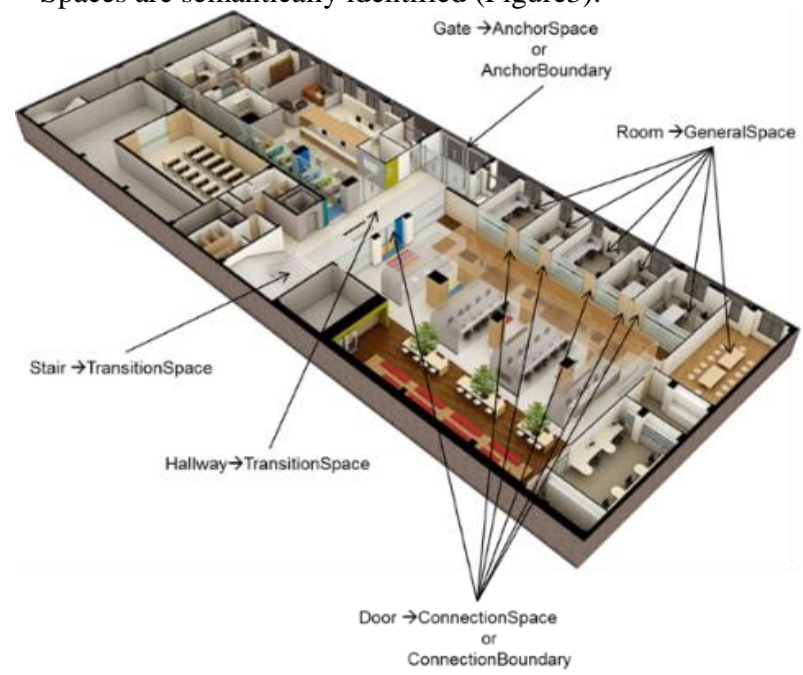

Figure 3: Semantics of the Indoor space in IndoorGML (Lee et al 2014)

\subsection{Node-Relation Graph}

The dual space is derived from the primal space applying Poincaré duality (Munkres, 1984). According to Poincaré duality, a k-dimensional object in N-dimensional primal space is mapped to (N-k) dimensional object in dual space. (Lee, 2004). In 3D primal space, 3D objects are mapped as 0D objects (nodes) and 2D objects are mapped as 1D objects (edges) in 2D dual space. Thus, the dual space can be used to derive the NRG that will be used for path computation.

The NRG can be derived from a subset of the indoor space but also by applying different relationships between the cells (adjacency, connectivity, accessibility and so on). For example, non-navigable indoor cells do not participate in the composition of the NRG. Besides the indoor space can be subdivided. A cell 'room' can be split in smaller cells such as 'fax machine corner', 
'printer corner', and so on. Figure 4 illustrates this concept with four cases. In the figure: W stands for Walls, D for Doors and S for spaces, red lines represent edges in dual space, nodes are not shown. For clarity the drawings are in 2D. The first two examples show the influence of the door representation (2D or 3D). If the 'doors' are represented as surfaces (2D objects), they are mapped to edges (1D objects) in NRG (Figure 4a). NRG is more complex if 'doors' are solids (3D objects); they are then mapped to nodes (1D object) (Figure 4b). It is also possible to select which cells can be used for NRG. The third case shows an example of NRG where room cells are not taken into consideration. The resulting NRG connects only the door cells. The final case illustrates the above mentioned subdivision of an architectural element (e.g. room) on smaller units to ensure that each cell has access to only one door.

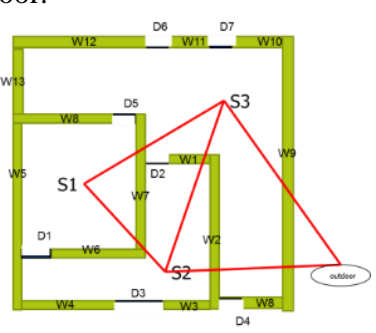

a)'thin' doors

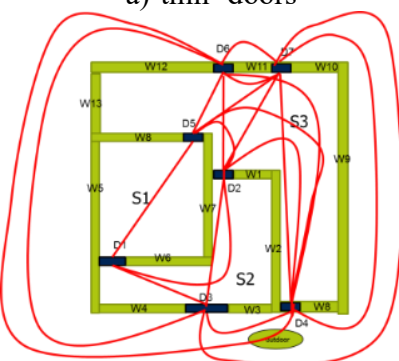

c)only door cells are used

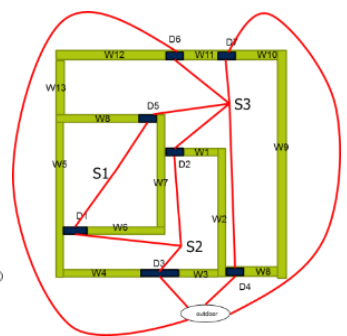

b) 'thick' doors
Figure 4: Examples of networks for navigation

Clearly, this flexible mechanism to extract a network for navigation by only modifying the cells in the primal space is the most notable characteristic of IndoorGML.

\section{LADM}

Land administrations systems (land registry, cadastre) have different origins in different countries. The information for land registry is sometimes collected for taxation purposes (Simpson, 1976, FIG, 199, Isikdag et al., 2015) and in other cases for legal security. Over the years, in many countries the land administration systems more and more served both applications; e.g. in the area of spatial development or spatial planning. In this context the term multi-purpose cadastre is used. Based on the initiative of the FIG (International Federation of Surveyors), ISO has developed the standard Land Administration Domain Model (LADM), ISO 19152:2012. In the standard, land administration is described as the process of determining, recording and disseminating information about the relationship between people and land (or rather 'space'). These recognised rights are in principle eligible for registration, with the purpose being to assign a certain legal meaning to the registered right (e.g. a title). Therefore, land administration systems are not just 'handling geographic information', as they represent a lawfully meaningful relationship among people, and between people and land. The model has been used by several institutions in different counties (http://isoladm.org/).

\subsection{LADM content and model}

The LADM standard defines a basic administrative unit ('basic property unit') as an administrative entity, subject to registration (by law), or recordation, consisting of zero or more spatial units ('parcels') against which (one or more) unique and homogeneous RRRs (rights, e.g. ownership right or land use right, responsibilities or restrictions) are associated to the whole entity, as included in a land administration system. A 'spatial unit' (Lemmen et al., 2010, ISO 19152, 2012) is a single area (or multiple areas) of land and/or water, or a single volume (or multiple volumes) of space. A spatial unit can be described by 2D or 3D geometry or even by textual descriptions (Lemmen at al., 2015). Homogeneous' means that a right, restriction or responsibility affects the whole basic administrative unit. 'Unique' means that a right, restriction, or responsibility is held by one or more parties (e.g. owners or users) for the whole basic administrative unit. Making the unit any larger would result in the combination of rights not being homogenous. This is by definition impossible, with the exception that two neighbours have equal RRRs and parties. Making the unit smaller would result in at least 2 neighbour parcels with the same combinations of rights. The spatial units are called legal or virtual objects, because they do not need to be visible in the real world.

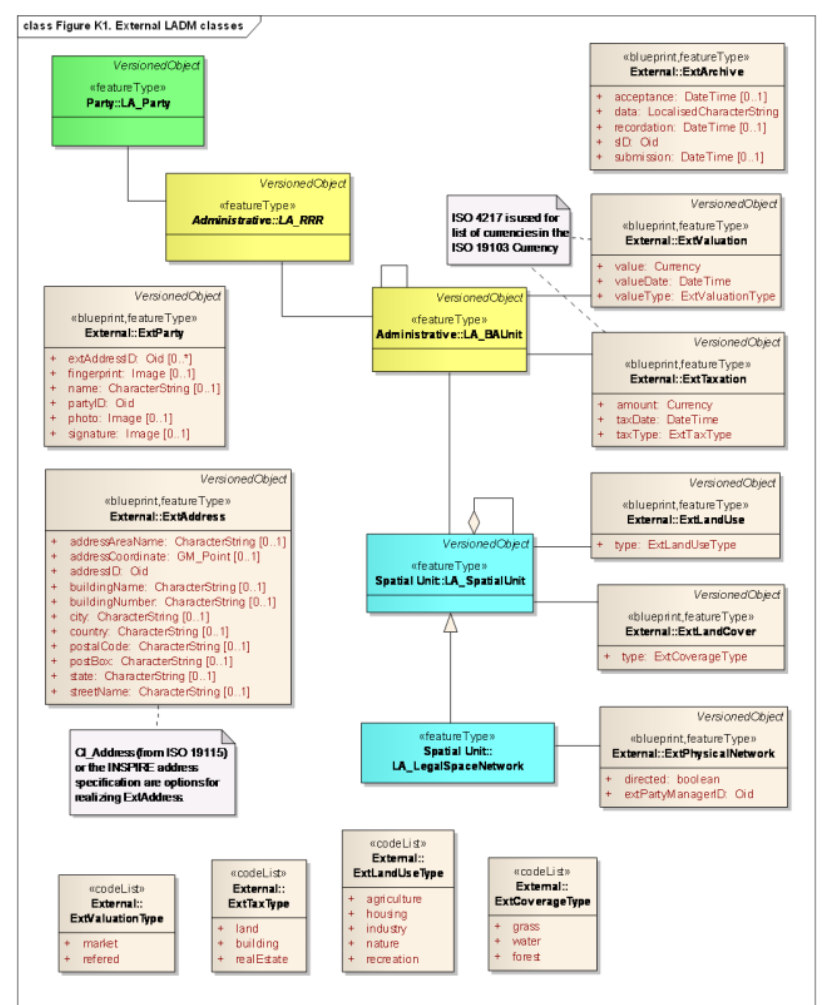

Figure 5: Core class LADM (in colour and) and LADM external classes (with 'Ext' prefix), taken from ISO 19152:2012

However, it should be noted that quite often the boundary of a parcel coincides with a physical real world object; e.g. a fence, wall, edge of road. In case of 3D spatial units, this is even more true; e.g. the geometries of physical objects such as tunnels, building (parts) or other constructions correspond also to legal spaces with unique and homogeneous RRRs attached (Lemmen et al 2010, Zulkifli et al 2013). Perhaps indoor navigation itself is not directly a 3D cadastre topic, but is it strongly related, because there is significant overlap between the indoor spaces as used in navigation and the $3 \mathrm{D}$ spatial units for registering the 
RRRs (in the environment of apartment and other buildings and constructions).

The main characteristic of the LADM can be summarised as follows:
- The basic classes of the model are 1) parties (people and organizations); 2) basic administrative units, rights, responsibilities, and restrictions (ownership rights); 3) spatial units (parcels, and the legal space of buildings and utility networks) and 4) spatial sources (surveying), and spatial representations (geometry and topology), (Figure 5).
- The spatial units are abstract spaces. They are geometric/ topological representation of rights and administrative units. Spatial unites can coincide with topographic features. LA_LegalSpaceNetwork is used to define rights for utility networks (not to be confused with navigation network as in IndoorGML)
- Spatial units can be unbounded.

\subsection{Relationship between physical and virtual objects}

A (3D) building registration is something else than a (3D) Cadastre. Cadastre is about the legal spaces. That is, spaces described by geometry (and topology) where certain RRRs are attached to. So, all kinds of building details, such as different rooms/ spaces, may not always be relevant from legal perspective (when same RRRs apply), but can always be defined. Only when the RRRs are different, a separate geometry is needed. So, most likely only a part of the indoor building modelling information may be relevant in 3D Cadastre context (and perhaps that geometry is even implicit; e.g. a 3D boundary face, defined by the 'middle of the wall'). The geometries of the real world (physical) objects and the geometries of the legal objects should be consistent and we should design rules for this.

The Annex K from ISO 19152 (Figure 5), is a UML diagram showing in colour core classes of the LADM standard: green, LA_Party (person), yellow, LA_RRR (right, etc. such as ownership)/LA_BAUnit in blue, LA_SpatialObject (parcel) and showing not in colour the LADM external classes (with stereotype <<blueprint>>, e.g. ExtTaxation, ExtValuation). LA_SpatialObject has several specializations, such as LA_LegalSpaceNetwork (shown in diagram, including link to ExtNetwork, the physical network registration) and LA_LegalSpaceBuildingUnit (not shown in diagram, but could be linked to physical building registration). LADM is more a conceptual framework defining concepts and terminology, than prescriptive standard. A country should first develop an LADM country profile supporting the legislation of the country (and described in concepts of the international standard), before transforming this into a land administration implementation.

\section{INDOORGML AND LADM: SYNERGIES}

The two standards have been developed for different purposes (navigation vs. land administration) and have different scope (indoor vs. indoor/outdoor, above/below surface). Therefore, they have many difference but also similarities. We will compare the space characterising of the two models and will explore a number options to combine the models; e.g.

- a formal approach for deriving a LADM space layer within IndoorGML context,
- an 'equivalence' association between LADM LA_SpatialUnit and IndoorGML abstract space for rights (RRRs), similar to other associations of LADM classes and other external classes.

Another aspect to be explored is that fact that IndoorGML contains 3D topographic information (in a way similar to LandXML, InfraGML, CityGML, BIM/IFC). 3D legal spaces often need reference objects to make sense (for orientation and understanding purpose). 3D legal space can have their own independent geometry and topology. Similarly IndoorGML allows subdivision and aggregations of spaces based on some properties (such as accessibility, security, etc.). However it is also possible to refer to topographic elements as some of the 3D space boundaries, or even refer to complete topographic spaces for the definition of LADM's legal spaces (which could then also contain operations, such as buffer of a 3D space, or a middle of a $3 \mathrm{D}$ wall/floor space to be assigned to neighbour room space, or aggregating a number of room spaces, etc.). The possible link (and operations on 3D topographic boundaries and spaces) between the topographic and LADM layer will be discussed in detail.

\section{Similarities:}

- Both models (can) deal with semantically annotated 3D spaces, which have properties.

- Both models operate with abstract spaces. Abstract spaces in IndoorGML can be defined on the basis of user or environment properties. Abstract spaces in LADM are based on legal regulations. Similarly, IndoorGML allows subdivision and aggregations of spaces such as accessibility, security, etc. The same is true in LADM: legal spaces can be grouped and organized in a hierarchy.

- Both models have a notion of primal space with geometry and topology. The 3D partitioning of LADM can be seen as primal space. LADM maintains links to external classes of which some are mentioned in annex $\mathrm{K}$ of the standard: building units, utility networks. IndoorGML provides links to CityGML, IFC and KML.

- Both models maintain several subdivisions of space. The mechanism in IndoorGML is by defining specific space layers. LADM abstract subdivisions are embedded in the conceptual schema (and called LA_Level).

- Both models maintain relationships between objects. LADM supports extensive set of relationships and constrains. Spatial relationships can be based on topology but could be also without topology (just geometry or even textual descriptions). IndoorGML does not have specific notions of constraints between objects, but rather topological relationships (i.e. adjacency and connectivity) is used to derive the dual space.

\section{Differences:}

- LADM is only a conceptual schema, while IndoorGML has XML implementation.

- IndoorGML requires non-overlapping subdivision of spaces, LADM may have overlapping abstract spaces but spatial units related to full ownership may not overlap with each other (but these might overlap with a spatial unit related to a certain restriction; e.g. because of an environmental protection zone).

- IndoorGML maintains primal and dual space, while LADM has only primal space.

- LADM models legal and administrative concepts ownership rights of spaces related to certain parties. IndoorGML might use such rights to specify subdivision, but no explicit Space Layer have been developed so far. 
Table 1: IndoorGML and LADM: a comparison

\begin{tabular}{|l|l|l|}
\hline Criteria & IndoorGML & LADM \\
\hline UML model & + & + \\
\hline XML schema & + & - \\
\hline Indoor space & + & + \\
\hline NRG & + & - \\
\hline Topographic space & + & + \\
\hline Outdoor space & - & + \\
\hline 3DGeometry & + & + \\
\hline Space subdivision & + & + \\
\hline Non-overlapping spaces & + & $+/-$ \\
\hline Unlimited spaces & - & + \\
\hline Space Layers & + & + \\
\hline Constraints & - & + \\
\hline Adjacency & + & + \\
\hline Connectivity & + & - \\
\hline Other topological relationships & - & + \\
\hline Legal/administrative aspect & - & + \\
\hline & & \\
\hline
\end{tabular}

For the purpose of IndoorGML, LADM can be used to define a framework for space subdivision. As mentioned above, if topological spatial units are defined, there are neither gaps nor overlaps in the partition in LADM. In this process the rights, restrictions and responsibilities as well as some administrative units play a critical role. The following example provides an illustration (Figure 6). Let us assume that the two-storey house belongs to Jim. He has rented the ground floor to Tim. The entire ground floor is used by Tim, but the stairs are shared by both. The indoor space will be the one shown in Figure 1. But Tom and Jim has different rights and therefore different cells will be used to create NRG, which can serve each of them. If NRG has to be provided for Tim, only the cells of the ground floor will be used. (Figure 6, upper left corner). If the NRG is intended for Jim, the cells of the upper floors and the cells representing the stairs have to be taken into consideration. Alternatively, if a renovation of the interior of both floors has to be performed all cells will be used to construct NRG.

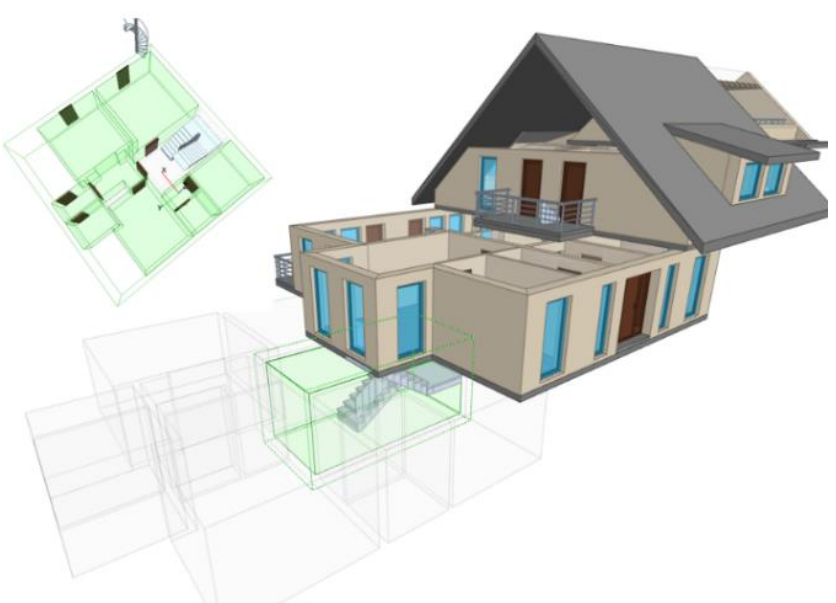

Figure 6: Example of space subdivision according to different property rights

In this example, there are two abstract subdivisions according to LADM: one on the basis of rights (an indoor space used by Tim and one used by Jim) and one on the basis of ownership (Jim owns the house). According to IndoorGML the indoor cells are defined with respect to the architectural elements of the house. However, for deriving NRG for Tim and Jim, the IndoorGML space needs to be enriched with information about the spatial units of LADM (as defined by responsibilities). The NRG for a maintenance team will most probably require information about the spatial unit of LADM that defines the ownership (the house of Jim). In this example, the spatial units of LADM can be seen as aggregation of some spaces of IndoorGML.

The following cases might be possible:

1) While IndoorGML has more detailed subdivision, LADM provides larger spatial units but follow the borders of IndoorGML units (this would be considered to most 'normal' scenario). IndoorGML inherits properties of LADM spatial units. In principle, LADM can have spatial units that have same RRR and Party. A possible option can be to adapt the LADM spatial units to IndoorGML spaces.

2) IndoorGML has less detailed subdivision. LADM imposes abstract borders across large rooms. Examples of such subdivision can be observed in airports or shopping malls. In that case the subdivision of IndoorGML has to be further subdivided and adapted to the legal units.

3) The two space subdivisions do not match without clear overlap of borders. Two options can be investigated: either subdividing to the smallest possible unit or using the IndoorGML spaces and providing properties as in case 1 . Such cases would commonly appear in big shopping malls with many overlapping rights.
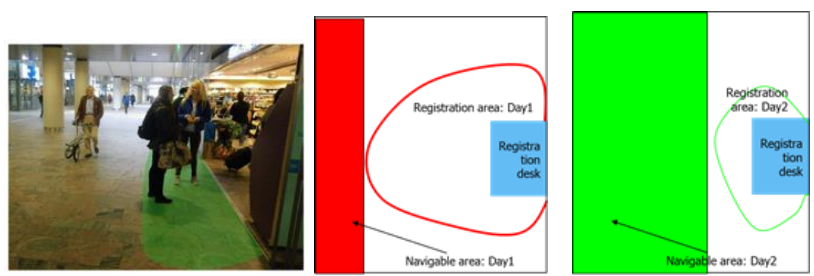

Figure 7: Abstract area delineated on the basis of function/use of the space. The function/use can change dynamically

4) Theoretically, IndoorGML might be used for dynamic space subdivision. LADM also supports temporal right (including cyclic and reoccurring patterns).

- IndoorGML may impose space subdivision (due to function or a user use), e.g. into 'coffee corner' or 'waiting area'. It might be then possible that a LADM spatial unit crosses these areas. It could be that the rented area for the shop (spatial unit) differs from the perceived navigation area (as in Figure 7). It is a case similar to case 3 , but considering the time component.

- IndoorGML imposes space aggregation (due to function or user use) as in Figure 2. LADM remains the same and crosses the new IndoorGML spaces.

In order to represent this complex relation between the two models, we envisage two options:

- option 1: an association relation between LADM LA_SpatialUnit (with respect to rights) and IndoorGML Cell Space (navigable and non-navigable), and

- option 2: LADM space layer within IndoorGML context.

The first option may be simply to insert an external link for each navigable space of IndoorGML to a spatial unit in LA_Spatial_Unit package of LADM and vice-versa without modifying IndoorGML and LADM. However, a single navigable space in IndoorGML can correspond to multiple spatial units of LADM and it is no longer possible to apply the first option with a one-to-one association. However, a one-to-many (normal case) or many-to-many association would be a way to model this or to subdivide the spatial units in LADM. Alternatively, it could be 
possible to specify a constraint that each IndoorGML Space could be defined in terms of spatial unit of LADM to maintain one-to-one correspondence. However, this feels less appropriate given the main purpose of IndoorGML.

The second option is to provide an extension module of IndoorGML to interface IndoorGML and LADM. This extension includes the spatial unit package of LADM, where each object of spatial object in this module has an external link to an object in the basic administrative unit in LA_BAUnit of LADM. For this option, it will be needed to convert LA_Spatial_Unit of LADM to an extension module of IndoorGML.

\section{SUMMARY AND FUTURE RESEARCH}

This is the first attempt to find synergies between the two standards IndoorGML and LADM. This synergy is of great importance for IndoorGML, as right would provide useful information for identifying navigable spaces for different users. The paper highlighted the most important characteristics of the two standards and a number of use cases. The analysis of the two standards shows differences but also many similarities, which we believe will make it possible to establish a link. The analysis has clearly shown that a link can be established via the spatial unit of LADM and the space of IndoorGML. The mechanism of establishing the link needs further elaboration. The two options direct link or LADM space layer have advantages and disadvantaged that will be carefully studied in follow up papers.

\section{ACKNOWLEDGEMENTS}

The authors acknowledge the standardisation organisations OGC and ISO hosting the IndoorGML and LADM standards.

\section{REFERENCES}

Afyouni, I., C. Ray, and C. Claramunt, 2012, Spatial models for context-aware indoor navigation systems: A survey, Journal of Spatial Information Science, Number 4 (2012), pp. 85-123

Becker, T., C. Nagel, T.H. Kolbe, 2008, A Multi-layered SpaceEvent Model for Navigation in Indoor Spaces. In: Lee, Zlatanova (eds.). 3D Geo-Information Sciences, Lecture Notes in Geoinformation and Cartography, 2009, Part II, 61-77.

Brown, G., C. Nagel, S. Zlatanova and T.H. Kolbe, 2013, Modelling 3D Topographic Space Against Indoor Navigation Requirements, Progress and New Trends in 3D Geoinformation Science, LNG\&C, Springer, Heidelberg, New York, Dordrecht, London, pp. 1-22

Bandi, S. and D. Thalmann, 1998, Space discretization for efficient human navigation, Wiley Online Library.

Fallah, N., Apostolopoulos, I., Bekris, K., and Folmer, E., 2013. Indoor Human Navigation Systems: A Survey. Interacting with Computers, 25(1), 21-33.

buildingSMART International, 2013, IFC (Industry Foundation Classes) 4, http://www.buildingsmart-

tech.org/specifications/ifc-releases/summary

FIG, (1995), Statement on the Cadastre. Copenhagen, Denmark, International Federation of Surveyors (FIG): 17 p. ISBN 0-6444533-1
Goetz, M., and Zipf, A. 2011. Formal definition of a user adaptive and length-optimal routing graph for complex indoor environments. Geo-Spatial Information Science, 14(2), 119-128

Isikdag U., M. Horhammer, S. Zlatanova, R. Kathmann and P. van Oosterom, 2015. Utilizing 3D Building and 3D Cadastre Geometries for Better Valuation of Existing Real Estate. In proceedings FIG Working Week 2015, Sofia, Bulgaria, 17-21 May 2015, 18p.

ISO 19152 (E), 2012, Geographic information- Land Administration Model (LADM), ISO 2012, ISO/TC 211 Geographic information/Geomatics, 128p.

Kikiras, P., Tsetsos, V., and Hadjiefthymiades, S. 2006. Ontology-based user modeling for pedestrian navigation systems. In ECAI 2006 Workshop on Ubiquitous User Modeling (UbiqUM), Riva del Garda, Italy.

Kruminaite, M. and S. Zlatanova, 2014, Indoor Space Subdivision for Indoor Navigation, ISA'14, Proceedings of the Six ACM SIGSPATIAL International Workshop on Indoor Spatial Awareness, pp. 25-31

Lee, J., 2004, A spatial access-oriented implementation of a 3-D GIS topological data model for urban entities. Geoinformatica, 8 (3), pp. 237-264

Lee, J., K.-J Li, S. Zlatanova, T.H. Kolbe, C. Nagel, T. Becker, 2014, OGC IndoorGML, OGC 14-0051r1, http://www.opengeospatial.org/standards/indoorgml\#downloads (15 May, 2016)

Lemmen, C.H.J., P.J.M.van Oosterom and R. Bennett, 2015. The Land Administration Domain Model, Land Use Policy, 49, 2015, pp. 535-545

Lemmen, C.H.J., , P.J.M. van Oosterom, R. Thompson, J. P. Hespanha and H. Uitermark, 2010, The Modelling of Spatial Units (Parcels) in the Land Administration Domain Model (LADM). In: Proceedings of the XXIV FIG International Congress 2010, April 2010, Sydney, 28 p.

Li, K.-J., 2016, InroorGML - A standard for indoor spatial modelling Int. Arch. Photogramm. Remote Sens. Spatial Inf. Sci., XLI-B4, 701-704, doi:10.5194/isprs-archives-XLI-B4-701-2016, 2016.

Munkres, J. R., 1984, Elements of Algebraic Topology, AddisonWesley, Menlo Park, CA, 1984

Van Oosterom, P.J.M. and C.H.J. Lemmen, 2015, The Land Administration Domain Model (LADM): Motivation, standardisation, application and further development, Land Use Policy, 49, 2015, pp. 527-534

Raubal, M., and M. Worboys, 1999, A formal model of the process of wayfinding in built environments. In Spatial information theory. Cognitive and computational foundations of geographic information science (pp. 381-399). Springer Berlin Heidelberg

Richter, K. F., Winter, S., and Ruetschi, U. J. 2009. Constructing hierarchical representations of indoor spaces. In Mobile Data Management: Systems, Services and Middleware, 2009. MDM'09. Tenth International Conference on Mobile Data Management Systems, 686-691, IEEE 
Simpson, S.R., 1976, Land Law and Registration. Cambridge University Press.

Sithole, G. and S. Zlatanova, 2016, Position, location, place and area: an indoor perspective, ISPRS Ann. Photogramm. Remote Sens. Spatial Inf. Sci., III-4, 89-96, doi:10.5194/isprs-annals-III4-89-2016, 2016

Worboys, M. 2011. Modelling indoor space. In Proceedings of the 3rd ACM SIGSPATIAL International Workshop on Indoor Spatial Awareness. 1-6. ACM

Zlatanova, S., L. Liu, and G. Sithole, 2013. A Conceptual Framework of Space Subdivision for Indoor Navigation. ISA '13 Proceedings of the Fifth ACM SIGSPATIAL International Workshop on Indoor Spatial Awareness, ACM New York, NY, USA. pp. $44-48$

Zlatanova, S., L. Liu, G. Sithole, J. Zhao and F. Mortari, 2014, Space subdivision for indoor applications, GISt Report, nr. 66, Delft, 201450 p.

Zulkifli, N.A., Rahman, A.A., Van Oosterom, P.J.M., 2013. Developing 2D and 3D Cadastral Registration System based on LADM: illustrated with Malaysian cases. In: Proceedings of the LADM Workshop, Kuala Lumpur, Malaysia, September 2013, 